\title{
The Deja Vu Theory from my perspective
}

\author{
Md Sadique Shaikh* \\ Department of Psychology, ISTM Mumbai, MS, India \\ *Corresponding author: Md Sadique Shaikh, Department of Psychology, ISTM Mumbai, MS, India
}

\begin{abstract}
You might be surprise as this article progress to read line after line with knowing the fact of Humankind and the connection between Human-Life and Déjà vu theory. The word "déjà vu "belong to the French words repository and the meaning is "Already Seen". Since several years déjà vu theory became a matter of debate among the space scientist and psychologist. The term belongs to human psychology but equally to space and universe domains as well. It's a reference of existence of "Parallel Universes" called "Multiverse". So, let us begin to the interesting write-up to know déjà vu occur when frequencies of two Universes match.
\end{abstract}

Keywords: Déjà Vu Theory; Parallel Universes; Multiverse; Human Psychology; Human Brain Quantum Psychology (Hbqp)

\section{Introduction}

Now let us begin with Déjà vu theory with my perspective and you decide your beliefs with your perspectives. Déjà vu is the French means "Already Seen" and the concept is already lived present situation/moment same as in past.i.e. several time we feel suddenly in the present moment of the life exactly same moment happened in the past and you strongly feel that you are in the same moment of past at present like function, event, seen, movement of life or whatever, and this occurrence called "Déjà vu occurred with you". The psychologist understand Déjà vu is just a mental illness/state/ sickness/illusion which occurred when majority circumstances of present moment strongly "Match" passed past moment of life. But I raised questions why those strongly match? why this happened? And how match? Where answer from psychology is uncertain. Now let me know why Déjà vu occurred and when Déjà vu occurred with reference of Quantum Mechanics and theory of Universe with integration of psychology called "Human Brain Quantum Psychology (HBQP). Let us start with the introduction, is many more universes exist in space and our Universe not alone one and the answer is Big YES. Several theories proved that our Universe not a single and like our Universe infinite parallel Universes exist in deep-space called "Multiverse" and every Multiverse has several earth-like and earth planets in it and each Earth existence of life in all Universes. Hence our life and entity not only limited on single earth of single universe but on all earths of all Universes (Multiverse). Now after separate discussion on Déjà vu and Multiverse theories (on the basis of Cosmic Microwave Background (CMB), theory of Black/ White holes, Dark Matter, String Theory, Quantum Mechanics and Androphobic principles). I would like to discuss what is the relation between Déjà vu theory and Multiverse theory, how Déjà vu prove existence of Multiverse and why Déjà vu occurred several times with all of us in in routine life with the help of below model (Figure 1).

Consider the number of parallel Universes in Multiverse U1 (Our Universe), U2, U3, U4, U5 ...... Uø tuned with different frequencies and moving with different revolution frequencies $\mathrm{F} 1$, F2, F3, F4, F5 ..............Fx and each parallel universe separated by cosmic walls hence invisible and no interference with one another, but when any two three or more Universes in Multiverse (Parallel Universes) during revolution tuned on the same frequency (inphase) or frequency match of two universes in my model you can see our Universe U1 tuned or frequency match or in-phase with same frequency F1 with other Universe U4 of Multiverse, hence caused weakened the cosmic walls to superimpose and interference on one another and Universes U1 and U4 connected, where each Universe has Earth with Life of all of us using thoughts frequency and Quantum Psychology life of several connect of Universe U1 with Universe U4 with our existence on all Universes Earths. It is possible our life in comparison one earth from other back or forth with light years and space time might be possible what moment we living right now or at present in Universe U1 Earth already we lived same moment on Earth of Universe U4 three years before or might be live after some days, month or years due to back and forth of our lives. Therefore, window of our lives opens other tuned Universe to our Universe and we felt we already seen or passed from this moment, situation, scene, etc. called Déjà vu. Hence in this way there is a strong relation between human psychology, Déjà vu and Multiverse. 


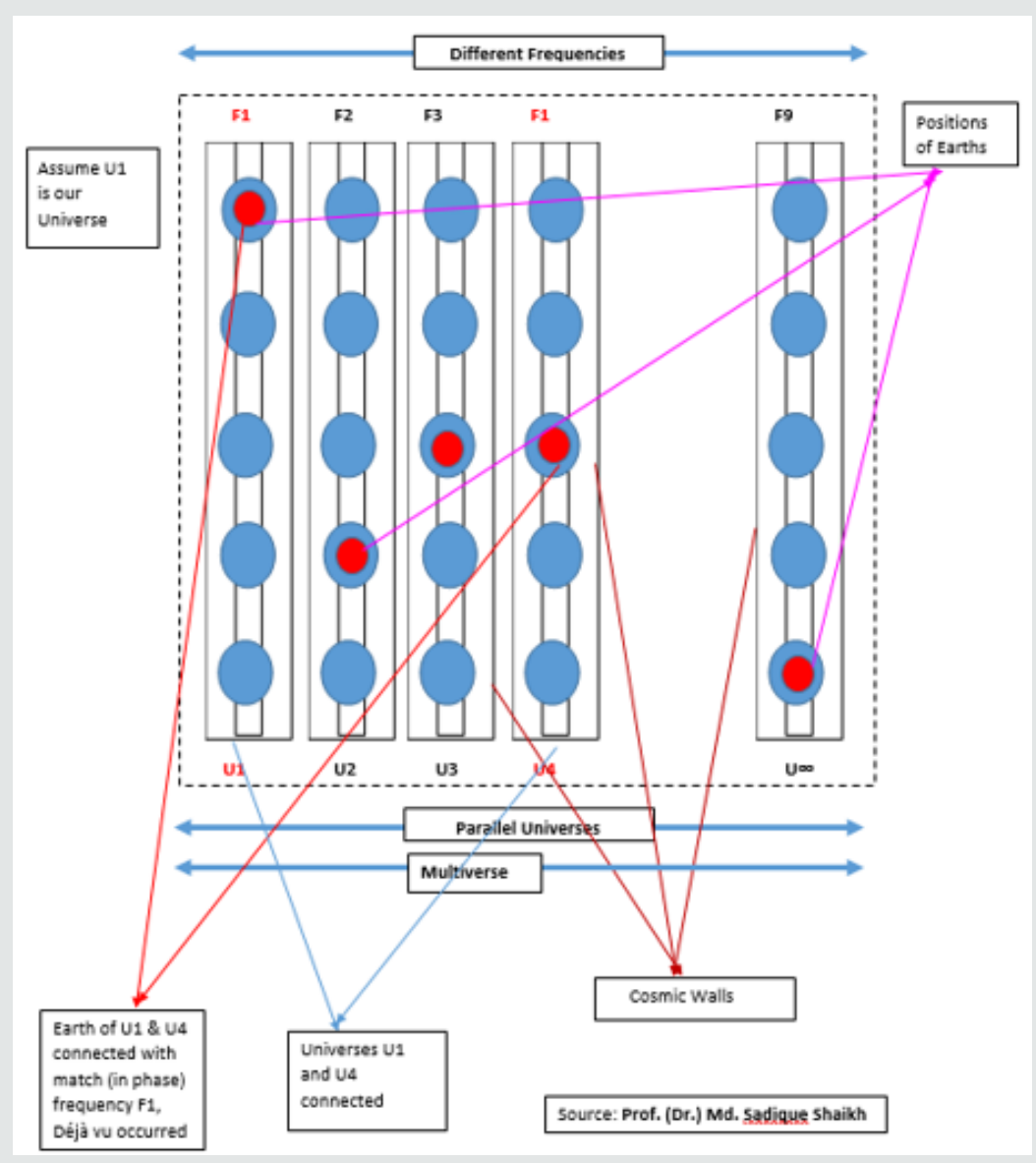

Figure 1: Different Revolution Frequencies and Parallel Universe.

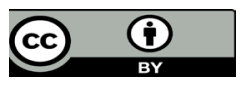

This work is licensed under Creative Commons Attribution 4.0 License

To Submit Your Article Click Here:

Submit Article

DOI: $10.32474 /$ SJPBS.2019.03.000152

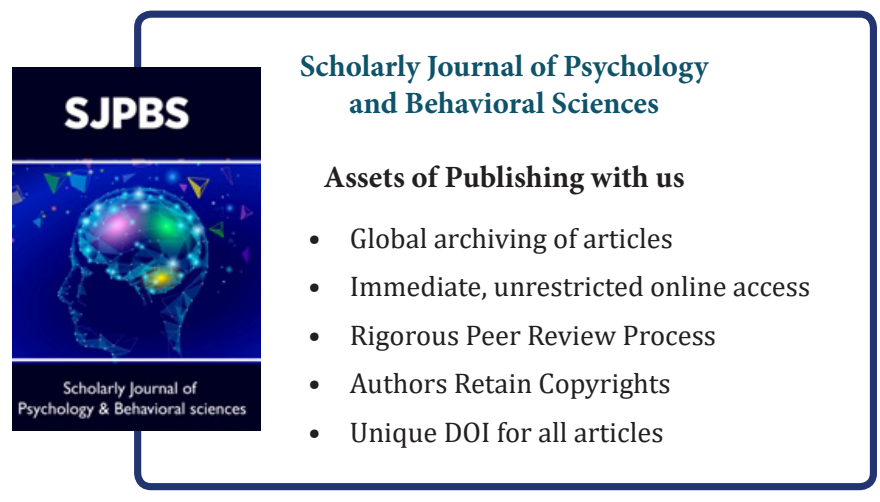

